\title{
Direct ionization and electron capture in $M$-shell x-ray production by fluorine ions
}

\author{
R. Mehta, ${ }^{*}$ J. L. Duggan, F. D. McDaniel, and M. C. Andrews \\ Department of Physics, North Texas State University, Denton, Texas 76203 \\ G. Lapicki \\ Department of Physics, East Carolina University, Greenville, North Carolina 27834 \\ P. D. Miller \\ Physics Division, Oak Ridge National Laboratory, Oak Ridge, Tennessee 37830 \\ L. A. Rayburn \\ Department of Physics, University of Texas at Arlington, Arlington, Texas 76019 \\ A. R. Zander \\ Department of Physics, East Texas State University, Commerce, Texas 75428 \\ (Received 2 May 1983)
}

\begin{abstract}
Measurements of $\boldsymbol{M}$-shell $\mathrm{x}$-ray production cross sections are reported for thin solid targets of ${ }_{79} \mathrm{Au},{ }_{82} \mathrm{~Pb},{ }_{83} \mathrm{Bi}$, and ${ }_{92} \mathrm{U}$. Fluorine ions of energies 25,27 , and $35 \mathrm{MeV}$ and charge states of 4, 5, 6, 8 , and 9 were used. The microscopic cross sections were determined from measurements made with targets ranging in thickness from $\sim 1$ to $\sim 300 \mu \mathrm{g} / \mathrm{cm}^{2}$. An enhancement in the target $M$-shell xray production cross section was observed for fluorine ions with one or two $K$-shell vacancies over those without a $K$-shell vacancy. The sums of cross sections for direct ionization to the target continuum and electron capture to the projectile's $L, M, N, \ldots$ shells are inferred from charge state $q=4,5,6$ data. The first Born calculations overpredict the cross-section data at all energies. Cross sections for electron capture from the target $M$ shell to the projectile $K$ shell for one $(q=8)$ and two $(q=9) K$-shell vacancies in the projectile are also overpredicted by the first Born approximation for electron capture, i.e., the Oppenheimer-Brinkman-Kramers approximation of Nikolaev. The data are in good agreement with the ECPSSR theory of Brandt and Lapicki, which accounts for the energy-loss, Coulomb-deflection, and relativistic effects in the perturbed-stationary-state theory.
\end{abstract}

\section{INTRODUCTION}

Direct ionization to the target continuum (DI) and electron capture to the projectile (EC) are the primary mechanisms involved in inner-shell vacancy production in heavy-ion-atom collisions. Two theoretical methods used in the present work to understand these processes are the first Born approximation and the ECPSSR (energy loss, Coulomb deflection, perturbed stationary state, with relativistic effects) method. In the first Born approach, the contribution of DI is calculated in the plane-wave Born approximation (PWBA) ${ }^{1}$ and the contribution of $E C$ is calculated in the Oppenheimer-Brinkman-Kramers (OBK) approximation of Nikolaev (OBKN). ${ }^{2}$ The ECPSSR approach, ${ }^{3}$ based on the perturbed-stationary state (PSS) formalism, ${ }^{4}$ goes beyond the first Born and takes into account energy loss, Coulomb deflection, and relativistic effects. The ECPSSR has recently been extended by Lapicki ${ }^{5}$ to $M$-shell ionization.

Studies by McDaniel and co-workers for $K$-shell ionization by ${ }_{14}^{28} \mathrm{Si}$ ions ${ }^{6}$ and for $L$-shell ionization by ${ }_{9}^{19} \mathrm{~F},{ }_{14}^{28} \mathrm{Si}$, and ${ }_{17}^{35} \mathrm{Cl}$ ions ${ }^{7}$ found enhancements in the cross sections for projectiles with $K$-shell vacancies over those without $K$-shell vacancies. This enhancement was successfully explained by the EC theory of Lapicki and McDaniel, ${ }^{8}$ based on the perturbed-stationary-state approach. The present work was undertaken to broaden the scope of these measurements to $M$-shell ionization and to make comparisons with currently available theories.

$M$-shell x-ray production for vanishingly thin targets of ${ }_{79} \mathrm{Au},{ }_{82} \mathrm{~Pb},{ }_{83} \mathrm{Bi}$, and ${ }_{92} \mathrm{U}$ were measured for $25-, 27-$, and $35-\mathrm{MeV}{ }_{9}^{19} \mathrm{~F}^{q+}$ ions. The charge states of the fluorine ions ranged from $q=6,8,9$ at $35 \mathrm{MeV}$ to $q=4,5,6,8,9$ at 25 and $27 \mathrm{MeV}$. The $M$-shell $\mathrm{x}$-ray production cross sections for projectiles with one or two $K$-shell vacancies are contrasted with the average $M$-shell $x$-ray production cross sections for projectiles without $K$-shell vacancies. Cross sections for DI plus EC to $L, M, \ldots$ shells and EC to the $K$ shell of the projectile are inferred and compared to the predictions of the first Born and ECPSSR theories.

\section{EXPERIMENTAL PROCEDURE AND DATA ANALYSIS}

The 25-, 27-, and 35-MeV ${ }_{9}^{19} \mathrm{~F}^{q+}$ ions were produced by the EN Tandem Van de Graaff accelerator at Oak Ridge National Laboratory. The ion beam was magnetically selected for charge states $q=4,5,6,8$, or 9 at 25 and 27 $\mathrm{MeV}$ and $q=6,8$, or 9 at $35 \mathrm{MeV}$. After collimation, the ion beam was incident on targets inclined at $60^{\circ}$ to the 


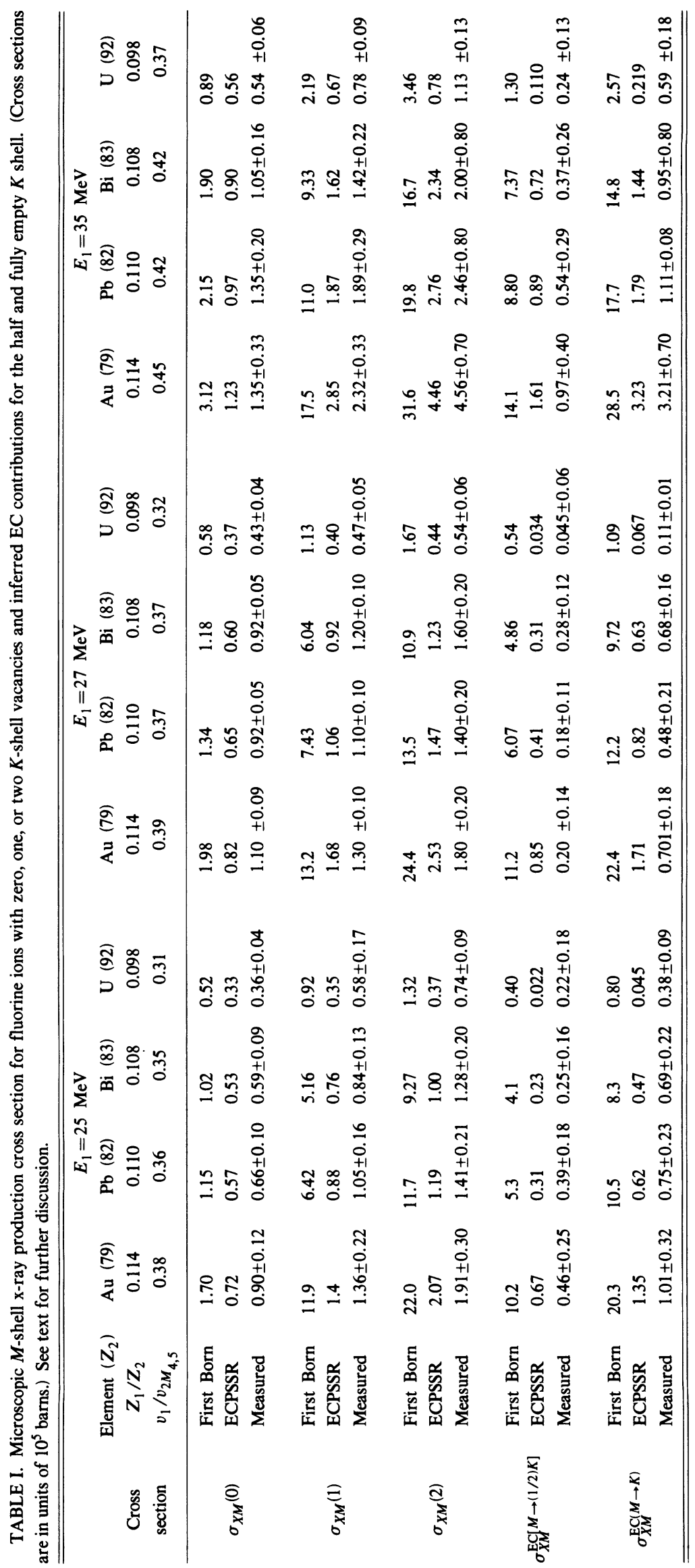




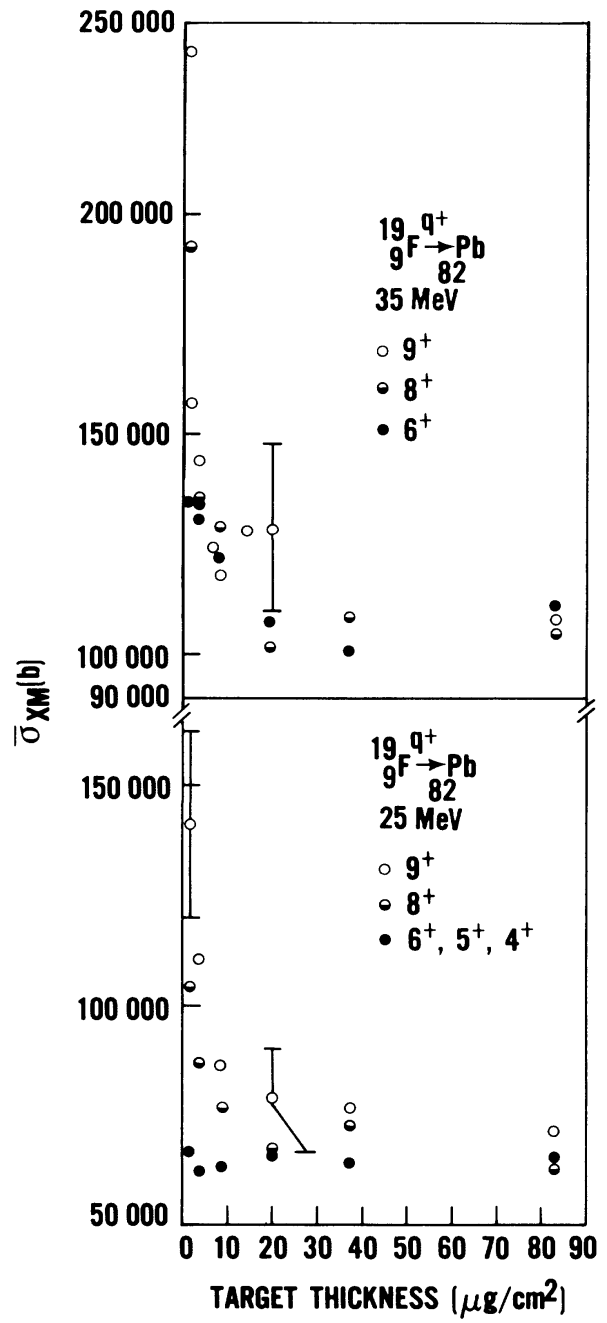

FIG. 1. Weighted average $M$-shell $\mathrm{x}$-ray production measurements $\bar{\sigma}_{X M}$ of $\mathrm{Pb}$ for $25-$ and $35-\mathrm{MeV}{ }_{9}^{19} \mathrm{~F}^{q+}$ ions vs the target thickness. Charge states of the fluorine ions were $q=4,5,6,8,9$ at $25 \mathrm{MeV}$ and $q=6,8,9$ at $35 \mathrm{MeV}$. Only the measurements for the thinnest targets $\left(\leq 1 \mu \mathrm{g} / \mathrm{cm}^{2}\right)$ approximate single-collision conditions and therefore microscopic cross sections.

beam. A Si $(\mathrm{Li})$ detector positioned at $90^{\circ}$ to the beam direction measured the $\mathrm{x}$ rays produced in the target. The scattered particles were detected by a silicon surfacebarrier detector at $30^{\circ}$ with respect to the incident beam direction.

The targets were made by vacuum evaporation and deposition of the elements $\mathrm{Au}, \mathrm{Pb}, \mathrm{Bi}$, and $\mathrm{U}$ on 15-20$\mu \mathrm{g} / \mathrm{cm}^{2}$ carbon backings. Methods were used to assure that the targets were $99.9 \%$ pure and nearly contaminant free. ${ }^{9} M$-shell x-ray spectra were measured for a range of thicknesses $\left(\sim 1\right.$ to $\left.\sim 300 \mu \mathrm{g} / \mathrm{cm}^{2}\right)$ for each element and for each charge state of the ions listed above in order to ascertain the effects of multiple collisions and to ensure that the data presented are for approximately singlecollision conditions. $M$-shell x-ray yields for the thinnest target for each element at the various charge states were normalized to the Rutherford scattered particle yields to determine microscopic $\boldsymbol{M}$-shell x-ray production cross sec- tions under single-collision conditions.

The efficiency of the $\mathrm{Si}(\mathrm{Li})$ detector was determined ${ }^{10}$ by (1) measuring the $\mathrm{x}$ rays emitted from calibrated sources of ${ }^{51} \mathrm{Cr}$, ${ }^{55} \mathrm{Fe},{ }^{57} \mathrm{Co},{ }^{65} \mathrm{Zn}$, and ${ }^{241} \mathrm{Am}$, (2) measuring the $K$-shell x-ray yields per scattered particle from thin Al and $\mathrm{Si}$ targets for an incident $\mathrm{F}^{4+}$ beam and dividing them by available experimental $K$-shell x-ray production cross sections for $\mathrm{Al}(K \times$ ray at $1.487 \mathrm{keV})$ and $\mathrm{Si}(K \times$ ray at $1.74 \mathrm{keV}$ ), respectively, ${ }^{11}$ and (3) calculating the attenuation of the $\mathrm{x}$ rays in the Mylar film, Be entrance window, Au contact layer, and the Si dead layer and normalizing the theoretical curve determined from the above attenuation calculation to a measured efficiency point at a higher x-ray energy. Details of the spectrum analysis and data reduction have been discussed elsewhere. ${ }^{10}$

\section{RESULTS AND DISCUSSION}

In Fig. 1, measurements of $M$-shell x rays, displayed as weighted average cross sections, for a range of target thicknesses of $\mathrm{Pb}$ for 25 - and $35-\mathrm{MeV}$ fluorine ions are shown. The circles used in this figure symbolically represent a fluorine ion without any $K$-shell vacancies (solid circle), with one $K$-shell vacancy (half-open circle) and two $K$-shell vacancies (open circle). As can be seen from the figure, the solid circles are essentially independent of target thickness and the ion charge states for $q=4,5,6$. The constancy of the cross sections for charge $(q=4,5,6)$ implies near independence of the $M$-shell x-ray production cross section with different numbers of $L$-shell vacancies for the ion energies and ion-target systems studied in this work. The enhancement in the $\mathrm{x}$-ray cross sections is greater for $q=9$ data (open circle) than for $q=8$ data (half-open circle) by approximately a factor of 2 .

The microscopic $M$-shell x-ray production cross sections are determined from the weighted average cross section for the thinnest target used, which represents approximately single-collision conditions. Table I lists the microscopic $M$-shell x-ray production cross section for $\mathrm{Au}$, $\mathrm{Pb}, \mathrm{Bi}$, and $\mathrm{U}$ for incident fluorine ions. The cross sec-

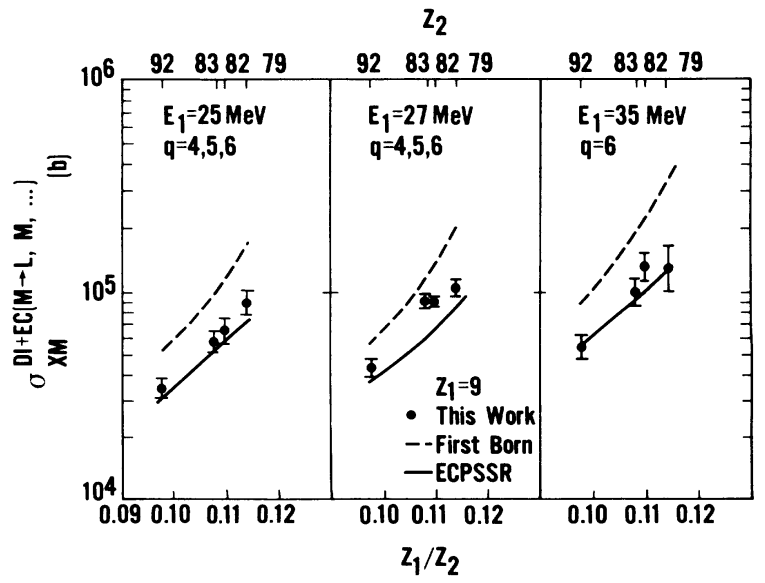

FIG. 2. Target $M$-shell $\mathrm{x}$-ray production cross sections $\sigma_{X M}^{\mathrm{DI}+\mathrm{EC}(M \rightarrow L, M, \ldots)}$ due to DI and EC to $L, M, \ldots$ shells of the projectile versus $Z_{1} / Z_{2}$ for $25-, 27-$, and $35-\mathrm{MeV}$ fluorine $\left(Z_{1}=9\right.$ and $\left.q \leq 6\right)$ with no $K$-shell vacancies. 

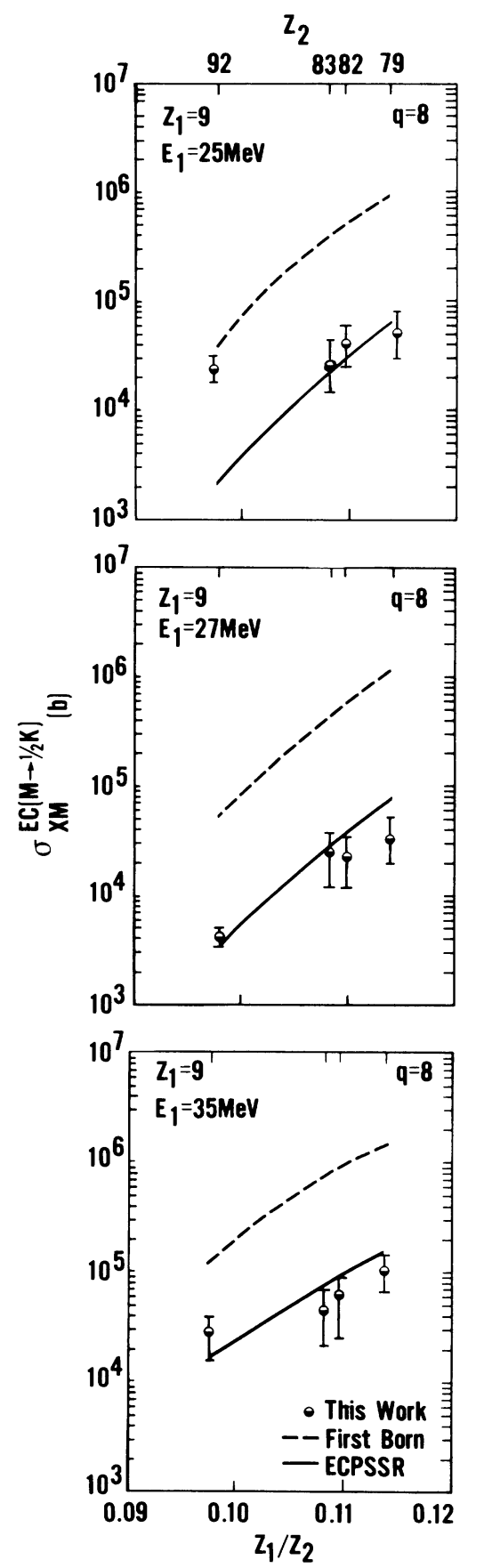

FIG. 3. Inferred target $\left(Z_{2}\right) M$ shell to projectile $K$-shell $\mathrm{x}$ ray production cross section due to $\mathrm{EC}, \sigma_{X M}^{\mathrm{EC}}{ }^{M \rightarrow(1 / 2) K]}$ for one $K$-shell vacancy $(q=8)$ vs $Z_{1} / Z_{2}$. Energies of the incident fluorine ions are 25,27 , and $35 \mathrm{MeV}$.

tions for $q=4,5,6$, which were identical within uncertainties, are presented as an average of the measured value for these charge states. Since $\boldsymbol{M}_{4}$ - and $\boldsymbol{M}_{5}$-subshell ionizations dominate the total $M$-shell ionization, a ratio of the velocity of the incident ion $v_{1}$ and the velocity of the target electron in the $M_{4,5}$ subshell, $v_{2 M_{4,5}}$, is a good indicator of the velocity ratio regime for our collision systems. The ratio $v_{1} / v_{2 M_{4,5}}=\left(E_{1} n^{2} / \lambda Z_{2 M}^{2} R\right)^{1 / 2}$ and is $\sim 0.3$ to 0.5 . Here, $E_{1}$ is the projectile energy (laboratory), $\lambda$ is the ratio of the projectile mass to the electron mass, $n=3$ is the principle quantum number, $R=13.6 \mathrm{eV}$ is the Rydberg constant and $Z_{2 M}=Z_{2}-21.15$ for $M_{4}, M_{5}$ subshells, with $Z_{2}$ being the target atomic number. In Table I, $\sigma_{X M}(0), \sigma_{X M}(1)$, and $\sigma_{X M}(2)$ are the microscopic $M$-shell $\mathrm{x}$-ray production cross sections for incident fluorine ions with zero, one, and two $K$-shell vacancies, respectively, with the subscript denoting cross sections of the $M$ shell. The inferred EC cross sections $\sigma_{X M}^{\mathrm{EC}(M \rightarrow K)}$ and $\left.\sigma_{X M}^{\mathrm{EC}} M \rightarrow(1 / 2) K\right]$ are determined by subtracting, correspond-

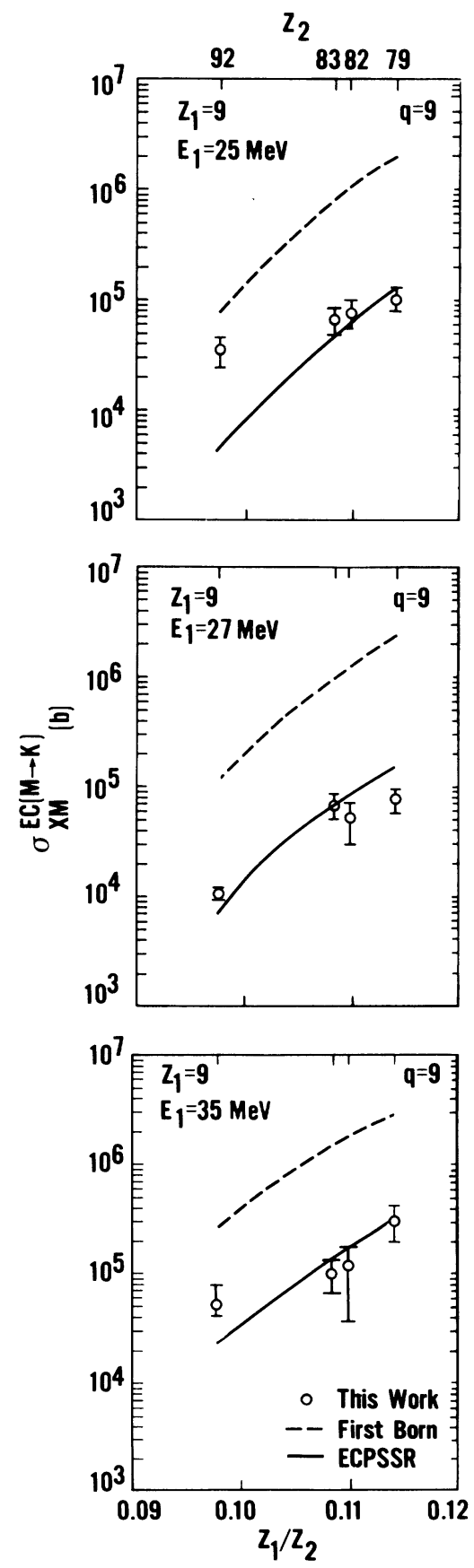

FIG. 4. Inferred target $\left(Z_{2}\right) M$-shell to projectile $K$-shell $\mathrm{x}$ ray production cross section due to $\mathrm{EC}, \sigma_{X M}^{\mathrm{EC}(M \rightarrow K)}$ for two $K$ shell vacancies $(q=9)$, vs $Z_{1} / Z_{2}$. Energies of the incident fluorine ions are 25,27 , and $35 \mathrm{MeV}$. 
ingly, $\sigma_{X M}(0)$ from $\sigma_{X M}(2)$ and $\sigma_{X M}(0)$ from $\sigma_{X M}(1)$. $\sigma_{X M}^{\mathrm{EC}(M \rightarrow K)}$ is the contribution to the $M$-shell $\mathrm{x}$-ray production cross section due to EC from the $M$ shell of the target to the $K$ shell of the projectile (for two $K$ vacancies present in the $q=9$ fluorine). $\sigma_{X M}^{\mathrm{EC}[M \rightarrow(1 / 2) K]}$ is the contribution to the $M$-shell x-ray production cross section due to the EC from the $M$ shell of the target to the $K$ shell of the projectile (for one $K$ vacancy in the $q=8$ fluorine).

In Table I, the theoretical cross sections are calculated in the framework of first Born approximation, i.e., PWBA for DI and OBKN for EC and the ECPSSR. Calculations are made for zero, one, or two $K$-shell vacancies in the projectile. The $M$-shell $\mathrm{x}$-ray production cross section $\left.\sigma_{X M}^{\mathrm{DI}+\mathrm{EC}(M \rightarrow L, M}, \ldots\right)$ for $q=4,5,6$ represents contributions due to DI and EC from the $M$ shell of the target to the $L, M, \ldots$ shells of the projectile and is given by $\sigma_{X M}(0)$ in the table.

The comparisons of the data with the theoretical predictions of first Born and ECPSSR approaches are shown in Figs. 2, 3, and 4. In all three of the figures, the first Born calculations are displayed by a dashed curve, while the ECPSSR calculations are represented by a solid curve. The theoretical ionization cross sections were converted ${ }^{12}$ to $\mathrm{x}$-ray production cross sections for this comparison, with the use of fluorescence yields and Coster-Kronig rates from McGuire. ${ }^{12}$

In Fig. 2, the first Born calculations overpredict the data at all energies. The ECPSSR theory shows good agreement with the data at 24 and $35 \mathrm{MeV}$ and underpredicts at $27 \mathrm{MeV}$. The constancy of data with charge states $q=4,5,6$ as seen in Fig. 1, and the good agreement noticed earlier ${ }^{13}$ in a comparison of experimental $M$-shell
$\mathrm{X}$-ray production cross sections and PWBA for DI suggest the EC to the $L, M$, and higher shells is not a large contributor to the total $M$-shell x-ray production cross section at these energies and for these ion-target systems.

In Figs. 3 and 4 , the inferred $\sigma_{X M}^{\mathrm{EC}[M \rightarrow(1 / 2) K]}$ and $\sigma_{X M}^{\mathrm{EC}(M \rightarrow R)}$ for $\mathrm{Au}, \mathrm{Pb}, \mathrm{Bi}$, and $\mathrm{U}$ are plotted for hydrogenlike $(q=8)$ and fully stripped $(q=9)$ fluorine ions, respectively, versus $Z_{1} / Z_{2}$. In both figures, the OBKN EC calculations overpredict the data. Good agreement is evident between predictions of the ECPSSR theory and the data, except for the ${ }_{92} \mathrm{U}$ data at $25 \mathrm{MeV}$. An underestimation of factors of $\sim 10$ and $\sim 5$ is noted at this energy for $q=8$ and 9 , respectively. The ECPSSR overpredicts the ${ }_{79} \mathrm{Au}$ and ${ }_{82} \mathrm{~Pb}$ data points at $27 \mathrm{MeV}$ to a lesser degree.

In conclusion, the ECPSSR theory provides much better estimate of the $M$-shell x-ray production cross section than the first Born theory. The EC is small for fluorine ions with no $K$-shell vacancies. The EC contribution to the total $M$-shell $\mathrm{x}$-ray production cross section is enhanced when the fluorine ion contains vacancies in the $K$ shell. This enhancement, within experimental uncertainties, is twice as large when, instead of one $K$-shell vacancy, two $K$-shell vacancies are available on the projectile.

\section{ACKNOWLEDGMENTS}

Work supported in part by the Robert A. Welch Foundation, the State of Texas Organized Research Fund, and the U.S. Department of Energy Contract No. W-7405ENG-26 with the Union Carbide Corporation.
*Present address: Department of Physics, University of Texas at Arlington, Arlington, TX 76019.

${ }^{1}$ D. E. Johnson, G. Basbas, and F. D. McDaniel, At. Data Nucl. Data Tables $\underline{24}, 1$ (1979).

${ }^{2}$ V. S. Nikolaev, Zh. Eksp. Teor. Fiz. 1ㅣ, 1263 (1966) [Sov. Phys._JETP 24, 847 (1967)].

${ }^{3}$ W. Brandt and G. Lapicki, Phys. Rev. A 223, 1717 (1981).

${ }^{4}$ G. Basbas, W. Brandt, and R. H. Ritchie, Phys. Rev. A I, 1971 (1973).

${ }^{5}$ G. Lapicki, Bull. Am. Phys. Soc. 26, 1310 (1981).

${ }^{6}$ F. D. McDaniel, J. L. Duggan, G. Basbas, P. D. Miller, and G. Lapicki, Phys. Rev. A 16, 1375 (1977).

${ }^{7}$ F. D. McDaniel, A. Toten, R. S. Peterson, J. L. Duggan, S. R.
Wilson, J. D. Gressett, P. D. Miller, and G. Lapicki, Phys. Rev. A 19, 1517 (1979).

${ }^{8}$ G. Lapicki and F. D. McDaniel, Phys. Rev. A 22 1896 (1980).

${ }^{9}$ P. M. Kocur, J. L. Duggan, R. Mehta, J. Robbins, and F. D. McDaniel, IEEE Trans. Nucl. Sci. NS-30, 1580 (1983).

${ }^{10}$ R. Mehta, J. L. Duggan, J. L. Price, F. D. McDaniel, and G. Lapicki, Phys. Rev. A 26, 1883 (1982).

${ }^{11}$ W. N. Lennard and D. Phillips, Nucl. Instrum. Methods 166 , 521 (1979).

${ }^{12}$ E. J. McGuire, Phys. Rev. A $\underline{5}, 1043$ (1972).

${ }^{13}$ R. Mehta, J. L. Duggan, F. D. McDaniel, M. C. Andrews, R. M. Wheeler, R. P. Chaturvedi, P. D. Miller, and G. Lapicki, IEEE Trans. Nucl. Sci. NS-28, 1122 (1981). 